\section{Measures towards success}

\section{Washlngton}

THIs year's award of the Nobel prize for physics, half going to Norman F. Ramsey of Harvard University and half shared by Hans G. Dehmelt of the University of Washington in Seattle and Wolfgang Paul of the University of Bonn, has its roots in the beginnings of quantum theory.

The technical accomplishments of all three prizewinners rely on the phenomenon, first recognized by Einstein, of stimulated emission, wherein an atom in an excited electronic state can be induced to fall to a state of lower energy by electromagnetic radiation of the frequency corresponding to the energy difference.

The prizewinners' innovations have been instrumental in testing the quantum theory of atoms with exquisite precision, and have also led to precise standards of measurement of general utility. The atomic clock, which depends on Ramsey's inventions, is now used to define one second of time as 9,192,631,770 oscillations of between hyperfine levels of caesium.

Ramsey's great achievement was the refinement of I. I. Rabi's method for inducing atomic transitions by passing excited atoms through a magnetic field and an electromagnetic radiation field of fixed frequency. Ramsey added a second radiation field, setting up an interference pattern with the first in which photons of the precise frequency needed to induce an atomic transition existed only in certain places. This restriction of the volume in which transitions could occur increased the coherence of the photons thus emitted.

A later development, by Ramsey and Daniel Kleppner, was the invention of the hydrogen maser, in which excited atoms are held in a suitably tuned cavity. The maser not only allowed direct highprecision studies of the structure of the hydrogen atom, but also became widely used as a reference source in, for example, interferometric techniques in radioastronomy. The accurate frequency standard provided by the hydrogen maser allows observed radio signals at physically separated radiotelescopes to be comPhysicists rewarded: Wolfgang Paul (left), Hans Dehmelt and Norman Ramsey. (AP.) pared, and from the differences highresolution radio maps are calculated.

In Ramsey's work and its offshoots, precision is obtained by persuading many identical atoms to emit radiation coherently. In the work of Paul and Dehmelt, the goal has been to trap single ions or electrons in a suitable arrangement of electromagnetic fields, so that their properties may be studied directly. Paul's work began in the 1950 s in Bonn, where he first invented methods to focus ions using multipolar magnetic fields (as is done in particle accelerators today), separate ions of different mass, and store them in traps of electric fields with a pervading radiofrequency radiation field.

The trap works by ingenious application of Einstein's rules for the emission and absorption of radiation. An ion moving towards a source of electromagnetic radiation can be excited to a higher level by picking up a photon of suitable frequency. But when it de-excites, it emits a photon whose frequency, with respect to the stationary apparatus, is slightly increased by the amount of the Doppler effect. The ion therefore emits a photon of higher frequency than that absorbed, and the extra energy is taken out of the ion's motion: in other words, it slows down. This principle works to cool ions, and to prevent them from moving out of the trapping volume.

Dehmelt and his colleagues developed a slightly different method, the Penning trap, which they used in 1973 to capture and hold a single electron. This has enabled Dehmelt's group to measure the electron's $g$-factor - the departure of its magnetic moment from the classical value - to an accuracy of a few parts in 1,000 million. Like Ramsey's work with the hydrogen maser, such measurements have tested (and so far confirmed) the correctness of the quantum theory of electrodynamics. The award of the Nobel prize to Ramsey, Paul and Dehmelt recognizes that physics, at bottom, is the art of measuring nature.

David LIndley

IMAGE UNAVAILABLE FOR COPYRIGHT REASONS
SCIENCE JOURNALS

Breaking the paper barrier

\section{New Delhl}

A 30-YEAR-OLD policy banning the publication of Indian editions of foreign science and technology periodicals in order to protect local publishers may soon be abandoned. The Indian Department of Science and Technology (DST) has been asked by the government to re-examine the issue, and has sent out a two-page questionnaire to a cross-section of the scientific community in India.

The present policy was instituted in the mid-1950s because the government feared that local editions brought out by foreign publishers would suffocate domestic journals. This was part of a general Indian policy to achieve self-reliance in science and technology that included import limitations and customs barriers to protect local technological products.

But five years ago the government made an about-turn, liberalizing the technology importation policy because it found that protectionism had led only to industrial stagnation. The government is now wondering if the publishing policy should be relaxed, for similar reasons: Indian publishing has not prospered, and the cost of importing foreign journais is an increasing burden.

The opinion poll is being carried out by Current Science, whose editor, $\mathrm{S}$. Ramaseshan, says there are two extreme views on the issue. Behind the restrictive policy lies the idea that publication of foreign science and technology journals in India could "undermine not only the efforts to build up our own journals, but even the formation of a cohesive and committed scientific community".

But the opposing view is that local editions of foreign journals are essential if India's scientific and technological activities are to be on a par with international standards. Competition, Rameseshan believes, would "improve the quality of our science and our journals".

Some 15,000 scientists are expected to participate in the opinion poll, and DST hopes to make its recommendations to the government by the beginning of next year. Respondents will be asked whether or not they want a revision of the policy, and to list up to ten foreign journals that they would like see published in local editions.

For the time being, the French magazine La Recherche is the only foreign scientific publication appearing in India in English, but the demand for foreign science journals in a country with over 1,200 research establishments and 125 universities is something that overseas publishers are not likely to ignore.

K.S. Jayaraman 